\title{
Pinning of magnetic skyrmions in a monolayer Co film on Pt(111): Theoretical characterization and exemplified utilization
}

\author{
Dusan Stosic, ${ }^{1,2}$ Teresa B. Ludermir, ${ }^{1}$ and Milorad V. Milošević, ${ }^{2 *}$ \\ ${ }^{1}$ Centro de Informática, Universidade Federal de Pernambuco, Av. Luiz Freire s/n, 50670-901, Recife, PE, Brazil \\ ${ }^{2}$ Departement Fysica, Universiteit Antwerpen, Groenenborgerlaan 171, B-2020 Antwerpen, Belgium \\ (Received 25 October 2017; revised manuscript received 19 November 2017; published 1 December 2017)
}

\begin{abstract}
Magnetic skyrmions are nanoscale windings of the spin structure that can be observed in chiral magnets and hold promise for potential applications in storing or processing information. Pinning due to ever-present material imperfections crucially affects the mobility of skyrmions. Therefore, a proper understanding of how magnetic skyrmions pin to defects is necessary for the development and performance of spintronic devices. Here we present a fundamental analysis on the interactions of single skyrmions with atomic defects of distinctly different origins, in a Co monolayer on Pt, based on minimum-energy paths considerations and atomic-spin simulations. We first report the preferred pinning loci of the skyrmion as a function of its nominal size and the type of defect being considered, to further reveal the manipulation and "breathing" of skyrmion core in the vicinity of a defect. We also show the behavior of skyrmions in the presence of an extended defect of particular geometry, that can lead to ratcheted skyrmion motion or a facilitated guidance on a defect "trail." We close the study with reflections on the expected thermal stability of the skyrmion against collapse on itself for a given nature of the defect, and discuss the applications where control of skyrmions by defects is of particular interest.
\end{abstract}

DOI: 10.1103/PhysRevB.96.214403

\section{INTRODUCTION}

Magnetic skyrmions are topological spin configurations that arise in the spin pattern of certain chiral magnets [1-3]. These novel objects have been observed in bulk magnetic materials with noncentrosymmetric crystal lattices [4-6] and in ultrathin magnetic films on surfaces with strong spinorbit coupling [3,7]. Skyrmions represent smooth magnetic configurations where the spin direction wraps around the unit sphere, due to competition between the exchange and the Dzyaloshinskii-Moriya interaction (DMI) [8,9]. They can either appear as isolated objects $[10,11]$ or form a closepacked lattice $[2,4,12,13]$, where the recent discovery of room-temperature skyrmions [13-15] in ultrathin magnetic multilayers has drawn considerable attention from the research community. The topology-related properties, the small size (down to a few nanometers) [16], and the very small electrical currents $[10,17,18]$ needed to displace skyrmions makes them promising candidates for information carriers in ultradense magnetic storage devices [19-23]. The possibility to create and destroy [3] individual skyrmions, as well as to manipulate their motion [17,24], is an important milestone toward the development of future spintronic applications.

Pinning plays a very important role in the mobility of skyrmions: imperfections in the magnetic material (such as impurity atoms, lattice defects, and geometrical constrictions) act as pinning sites and can result in an effective slowdown or even capturing of the skyrmion $[13,23]$. This could lead to artificial placement of pinning centers within the material for precise positioning of individual skyrmions, as was previously done for magnetic domain walls [25]. However, overly strong pinning is detrimental for the intentional movement of magnetic skyrmions, such as in racetrack memories. Skyrmion lattices have been moved through materials with defects by

\footnotetext{
*milorad.milosevic@uantwerpen.be
}

laterally applied currents $[17,26,27]$, and it was found that they can be pinned below a critical current density but remain undisturbed for larger currents [18]. For individual magnetic skyrmions several pinning mechanisms have been studied theoretically: local maxima in magnetic exchange [28,29], vacancies in the magnetic material [30], areas with higher magnetic anisotropy $[10,18]$, and more recently magnetic grains with varying anisotropy or DMI [31,32]. Recent experiments [33] have also considered interactions with atomic-scale defects and have identified preferred pinning positions for the skyrmion.

While crucial for the development of future spintronic applications, a proper understanding of how magnetic skyrmions pin to defects remains lacking. Reference [33] discusses the pinning positions for individual skyrmions, but neglects to provide an exact description and only considers the static situation. Other theoretical works $[10,18,28-30]$ have studied the dynamical behavior of skyrmions around a defect, but could not quantify the exact transition from a pinned to an unpinned state. There are also a number of works in the literature that have considered skyrmion behavior in periodic $[34,35]$ and random [36-38] pinning arrays, ratchet geometries [39], and linear defects [40-43]. However, these works typically assume a phenomenological model for pinning (effective parabolic potentials) rather than a microscopically justified one.

To obtain the proper description of the interaction of a skyrmion with a defect, an energetic consideration of the depinning is needed in the microscopic framework. Transitions from complex magnetic states such as pinned skyrmions are slow events on the time scale of spin switching, which makes direct dynamical spin simulations an impractical way to characterize pinning. Instead, the maximum along the minimum energy path, which provides an estimate of the activation energy of a depinning transition, can be used to quantify the stability of a pinned magnetic state. In that way, the description of single-spin-resolved transitions from pinned skyrmion state essentially becomes a problem of finding the 
path of minimum energy on the energy surface, and the corresponding evolution of the magnetic spin texture.

With this in mind, in this paper we revisit the pinning of a magnetic skyrmion by considering the minimum energy paths for transition from a pinned state, where the skyrmion is trapped in a potential well induced by the given defect, to an unpinned state, where the pinning forces are negligible and the skyrmion is free to move through the material. For the magnetic system to consider, we choose a single atomic layer of Co grown epitaxially on the $\mathrm{Pt}(111)$ surface. Using atomistic spin simulations we investigate the interaction of magnetic skyrmions with different kinds of atomic-scale defects. We find that the pinning strengths and preferred positions of the skyrmion depend strongly on the type of defect being considered, and on the ratio of skyrmion size to the defect size. The effective pinning potentials have complex functional forms, and cannot be described by bare parabolic functions as assumed in phenomenological models. We also show that magnetic skyrmions can be significantly distorted and/or strongly change size during the (de)pinning transition. Finally, we examine how certain linear and planar defect geometries can be exploited to develop devices involving selective optical and magnonic excitations, skyrmion guidance, and racetrack memories.

The paper is organized as follows. In Sec. II we provide a brief description of our theoretical framework and numerical simulations. Section III is devoted to the understanding of how magnetic skyrmions pin to different kinds of defects. Section IV examines manipulation and control of skyrmions by defects, with an outlook to some spintronic applications utilizing the defects. Our findings are summarized in Sec. V.

\section{MODEL}

In this work we consider a monolayer film of cobalt (Co) with perpendicular magnetic anisotropy on a platinum $(\mathrm{Pt})$ substrate. The magnetic monolayer is modeled by a hexagonal lattice structure with a lattice constant of $\zeta=2.51 \AA$, similar to the values found in Refs. [44]. We describe the magnetic material by a standard effective Hamiltonian

$$
\begin{aligned}
H= & -\sum_{\langle i, j\rangle} J_{i j} \boldsymbol{S}_{i} \cdot \boldsymbol{S}_{j}-\sum_{\langle i, j\rangle} \boldsymbol{D}_{i j} \cdot\left(\boldsymbol{S}_{i} \times \boldsymbol{S}_{j}\right) \\
& -\sum_{i} K_{i}\left(\boldsymbol{S}_{i} \cdot \hat{\boldsymbol{z}}\right)^{2}-\mu_{0} \sum_{i} \boldsymbol{\mu}_{i} \cdot \boldsymbol{H}
\end{aligned}
$$

within the Heisenberg model. $S_{i}=\mu_{i} / \mu$ is a threedimensional unit vector representing the orientation of the effective magnetic moment $\boldsymbol{\mu}_{i}$ localized on atomic sites, where $\mu_{i}=2.1 \mu_{B}$ and $\mu_{B}$ is the Bohr magneton. Similar values for the spin moment are found in thin films of Co on $\operatorname{Pt}(111)$ [45]. $J_{i j}$ is the effective nearest-neighbor exchange integral and $\boldsymbol{D}_{i j}$ is the DM vector arising from a three-site indirect exchange mechanism that couples two atomic spins to a neighbor atom with large spin-orbit coupling [46]. Such interface-induced DMI vectors are perpendicular to the unit vector $\hat{\boldsymbol{u}}_{i j}$ connecting $\boldsymbol{S}_{i}$ and $\boldsymbol{S}_{j}$ within the interface plane, namely, $\boldsymbol{D}_{i j}=D_{i j}\left(\hat{\boldsymbol{u}}_{i j} \times \hat{\boldsymbol{z}}\right)$. $K_{i}$ describes the uniaxial perpendicular magnetic anisotropy with $\hat{z}$ normal to plane. The last term in Eq. (1) is the Zeeman energy due to an external magnetic field $\boldsymbol{H}$ applied in the $\hat{\boldsymbol{z}}$ direction. Since the dipolar interaction mainly acts as a shape anisotropy [1], it is possible to include its effect by reducing the anisotropy constant in the Hamiltonian $[47,48]$.

The considered sample is delimited by periodic boundary conditions and the size of the unit cell is chosen such that an individual skyrmion is not affected by the periodicity of the system. Simulations are performed at zero temperature. The exchange stiffness $(J=29 \mathrm{meV}$ per bond), DM interaction ( $D=1.5 \mathrm{meV}$ per bond), and effective anisotropy $(K=$ $0.293 \mathrm{meV}$ per atom) are taken from experimental works on $\mathrm{Co} / \mathrm{Pt}$ samples, as listed in Ref. [48]. The ground state of the system has ferromagnetic (FM) ordering, but a Néel skyrmion with hedgehog configuration (in-plane magnetization pointing along the radial direction) represents a metastable state with energies that are comparable to those found in Refs. [47,48]. To stabilize the skyrmion, the system is numerically relaxed with a steepest-descent method after initializing a circle of spins (with radius fixed to $4 \mathrm{~nm}$ ) which point in the opposite direction of magnetization [10]. This hard circular domain ensures convergence to a metastable skyrmionic (Sk) state if such a solution exists, since the spin configuration must pass through it before reaching the FM state. An external magnetic field $\boldsymbol{H}$ is applied in the $-\hat{\boldsymbol{z}}$ direction (parallel to the skyrmion core) and used to control the size of the skyrmion. As shown in Fig. 1(a), the skyrmion can grow up to roughly $9 \mathrm{~nm}$ in diameter at a field of $H=380 \mathrm{mT}$ before it collapses to the uniform state. Figures $1(\mathrm{~b})-1(\mathrm{~g})$ reveal substantial variations in the various components of the energy across different applied magnetic fields.

Transitions between magnetic states can be described by minimum energy paths (MEPs). The nudged elastic band (NEB) method [49] and its variations [50-53] are commonly used to find MEPs of transitions in atomic systems. The method involves constructing a discrete representation of some initial path between two local minima on the energy surface, and then using an iterative algorithm to find the nearest MEP. The discretization points represent individual configurations involving the relevant parameters which are referred to as "images" of the system. These images form a discrete approximation to an otherwise continuous transition path and should be displaced during the optimization only in directions that are perpendicular to the path [54]. The key idea behind the NEB method is the projection (also termed "nudging") of the true force and the spring force using the tangent to the path. While the true force represents the negative of the energy gradient and can be included by the component perpendicular to the path, the spring force controls the distribution of images and has to be projected on the path tangent so as not to affect the transverse displacements of the images.

The NEB method has also been applied to several magnetic systems (see Ref. [54], and references therein). In many cases, such as in Refs. [55,56], these are rather simple systems where magnetic spins only rotate in a given plane. The method, however, suffers from convergence problems when applied to more complex magnetic transitions. This arises from the fact that the path tangent does not take into account the curved manifold of the configuration space of a magnetic 

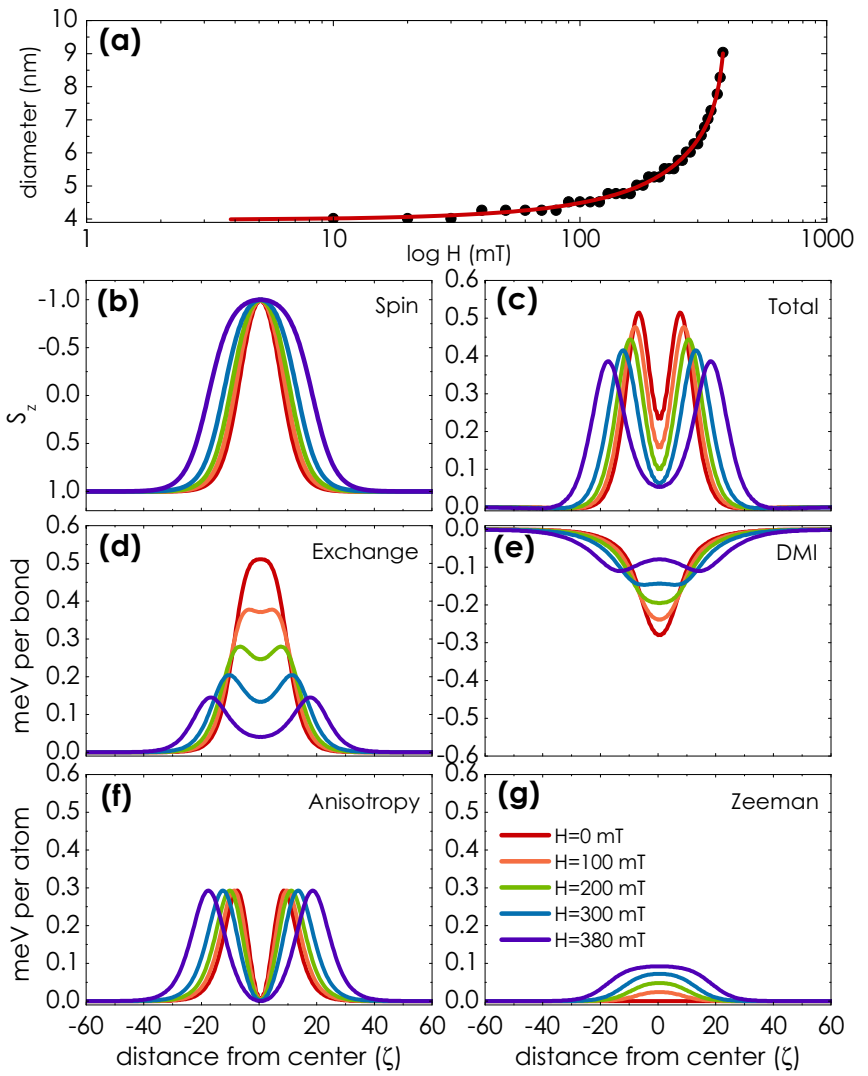

FIG. 1. (a) Core diameter in different external magnetic fields, and (b) line profile of magnetization $S_{z}$, for an individual skyrmion. Energy difference per Co atom or bond with respect to the uniform state for (c) total, (d) exchange, (e) DMI, (f) anisotropy, and (g) Zeeman contributions.

system due to constraints on the length of the magnetic vectors. Reference [54] tackles this problem by introducing the geodesic nudged elastic band (GNEB) with a geodesic measure of distances and projection of the path tangent as well as the forces on the tangent space of the curved manifold of magnetic configurations. The GNEB method can be applied to complex magnetic transitions involving nontrivial rotations of magnetic spins, such as skyrmion annihilation [47,48,54,57,58]. Reference [47] was the first to provide a detailed description of the collapse of an isolated skyrmion, finding two distinct mechanisms. However, it was later shown in Ref. [48] and proven in Ref. [59] that, in fact, only isotropic shrinking of the skyrmion (rotation of magnetic spins in the radial direction) represents a transition mechanism in the discussed Co monolayer on $\operatorname{Pt}(111)$. Reference [60] considered other paths to skyrmion collapse, such as that facilitated by the boundary. Finally, Ref. [57] extended the works on monolayer samples to few-atom-thick magnetic layers, and introduced spatially varied parameters as a pathway to an advanced control of the skyrmion stability.

In this paper we employ the GNEB method to estimate minimum energy paths for the discussed depinning transitions of a magnetic skyrmion. A total of 20 images are considered to form the elastic band, where the initial and final points are fixed to the pinned and unpinned states of the skyrmion on the defect, respectively. The remaining 18 images are energy minimized in a collective way using an iterative optimization procedure $[54,61]$. The initial path is constructed by relaxing the skyrmion with a single core spin that remains fixed at different positions along the sample, between the initial and final states. Given the complexity of the energy surface, different stable paths can be obtained depending on the initial guess. Convergence was verified by ensuring the energy gradient is sufficiently close to zero. After reproducing the test cases from Refs. [54] and [55], we simulated the monolayer skyrmion of Ref. [47] and recovered the same transition mechanism (and energy barriers) for skyrmion collapse as found in Ref. [48].

\section{PINNING FUNDAMENTALS}

Pinning at a defect can have different origins, and a theoretical analysis on the different ways to pin a skyrmion is needed. We therefore begin the discussion by considering pinning that arises from four different defects: local minimum of the exchange strength, increase of DMI, areas with lower magnetic anisotropy, and atomistic vacancies in the magnetic material. Physically, the local minima of exchange strength can be realized by engineering the density of itinerant electrons in the material $[29,62]$. One can alter DMI locally by increasing the interfacial spin-orbit coupling with two different materials below and above the film [14,15], and using lithographic techniques to change or remove the heavy-metal layer on top (see Refs. [57,63] for theory, and references therein for experimental realization). The strength of the magnetic anisotropy can be varied by distortions in the underlying atomic lattice and from impurity sites [26,64]. Lastly, the everpresent atomistic vacancies in ultrathin magnetic layers also exhibit pinning properties [30], but can be also strategically introduced and patterned to provide particular collective or individual manipulation of skyrmions [11].

\section{A. Pinning on material defects}

Defects due to spatial variations in material parameters (magnetic exchange, DM interaction, and anisotropy) are modeled by step functions (with hexagonal geometry) as shown in Fig. 2, where a set of lattice points is chosen to have different material parameter $M_{i j}$ from the rest of the sample that has $M_{i j}=M_{0}$. Figure 3 reveals that three distinct pinning positions can be effectuated depending on the properties of the considered defect. For anisotropy defects, the skyrmion pins at the domain wall when a lower anisotropy is considered $\left(K_{i}<K_{0}\right)$. A similar pinning behavior is found for inlayer defects with reduced exchange interaction $\left(J_{i j}<\right.$ $J_{0}$ ), where the parallel alignment of spins at the domain wall is favorable. Defects with increased DM interactions $\left(D_{i j}>D_{0}\right)$ favor canting of the magnetic spins and will therefore pin the skyrmion between the center and the domain wall where the angle between spins is the largest. Note that the pinning positions can be explained by individual energy contributions for a magnetic skyrmion (see Fig. 1). Defects with opposite material parameters to those discussed above will have their energy difference due to interaction with the skyrmion inverted, and will pin the skyrmion at its 


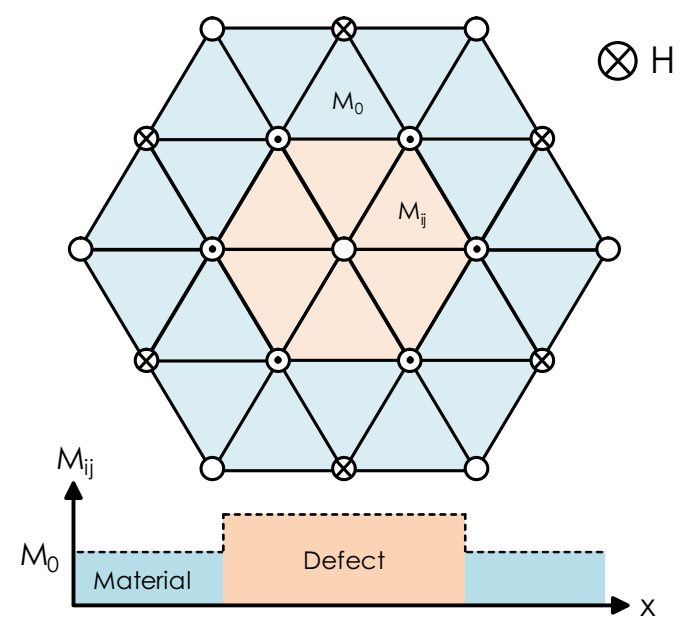

FIG. 2. Schematic illustration of the considered defects, having hexagonal geometry on a triangular lattice. Defects are represented by atoms (circles) and bonds (lines) inside the inner region (in red), while bonds intersecting the outer region (in blue) are removed in the case of hole defects (where atoms are absent). Bottom graph shows the line profile of the material parameter $M_{i j}$ (denoting either exchange, DMI, or anisotropy), being higher or lower inside the defect with respect to the rest of the sample where $M_{i j}=M_{0}$. Circles with dots correspond to nearest neighbors of the central lattice point, whereas circles with crosses represent next-nearest neighbors.

center. Table I summarizes the pinning positions of skyrmions at the considered material defects, with similar results to Refs. [24,29]. In the remainder of this paper we will consider defects with either $K_{i}=0.5 K_{0}, J_{i j}=0.9 J_{0}$, or $D_{i j}=2 D_{0}$, unless stated otherwise.

The preferred pinning position can also depend on the size of the defect with respect to the diameter of the skyrmion core. One might intuitively expect that a skyrmion with a large core will pin at a different location than a skyrmion with a small number of core spins. For a given size of the material defect, the skyrmion diameter can be controlled by an external magnetic field $\boldsymbol{H}$. Figure 4 reveals that an inlayer defect induced by variation in exchange or DM interaction

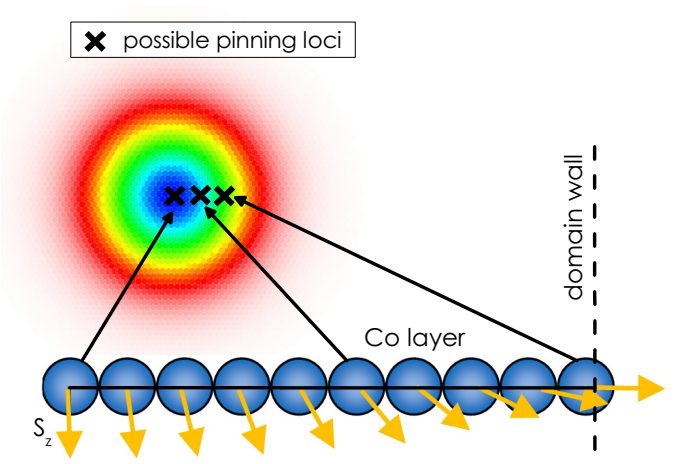

FIG. 3. Possible pinning positions for an individual skyrmion considering different kinds of defects. Spheres correspond to individual Co atoms. Skyrmions can pin either at the center (in blue), at the domain wall (in green), or between the two (in cyan) depending on the energy landscape in the vicinity of the defect.
TABLE I. Pinning position of a skyrmion at different kinds of material defects.

\begin{tabular}{|c|c|c|c|}
\hline Defect & $M_{i j}>M_{0}$ & $M_{i j}<M_{0}$ & Description \\
\hline Anisotropy & $\begin{array}{l}\text { At the center } \\
\text { of the core }\end{array}$ & $\begin{array}{l}\text { Off-center, } \\
\text { at domain wall }\end{array}$ & $\begin{array}{c}\text { Favors spins } \\
\text { normal to the plane }\end{array}$ \\
\hline Exchange & $\begin{array}{l}\text { At the center } \\
\text { of the core }\end{array}$ & $\begin{array}{l}\text { Off-center, } \\
\text { at domain wall }\end{array}$ & $\begin{array}{l}\text { Favors parallel } \\
\text { alignment of the spins }\end{array}$ \\
\hline DMI & $\begin{array}{l}\text { Between center } \\
\text { and domain wall }\end{array}$ & $\begin{array}{l}\text { At the center } \\
\text { of the core }\end{array}$ & $\begin{array}{l}\text { Favors canting } \\
\text { of the spins }\end{array}$ \\
\hline
\end{tabular}

would pin the skyrmion at its center for low magnetic fields (small skyrmions), but off-center for higher field values (large skyrmions). The off-center pinning occurs at the domain wall of the skyrmion core for exchange defects, and between the domain wall and the center of the skyrmion for DMI defects. The transition from center to off-center pinning can be explained by the alignment of magnetic spins in the skyrmion core. For large skyrmions with proper alignment of the core spins, a defect in the center of the skyrmion which reduces exchange or increases DMI will be unfavorable, whereas for small skyrmions such a defect is preferred precisely because it reduces the energy contributions due to canting of the spins. Figure 4 shows that the transition (ratio of skyrmion to defect size) is not linear and depends strongly on the character of the defect. DMI defects are far less likely to pin skyrmions off-center when compared to exchange defects, while both favor pinning at the center for larger defects.

Next we study the mechanism by which an isolated skyrmion transits from a pinned to an unpinned state. Figure 5 plots the minimum energy paths of pinning a skyrmion with

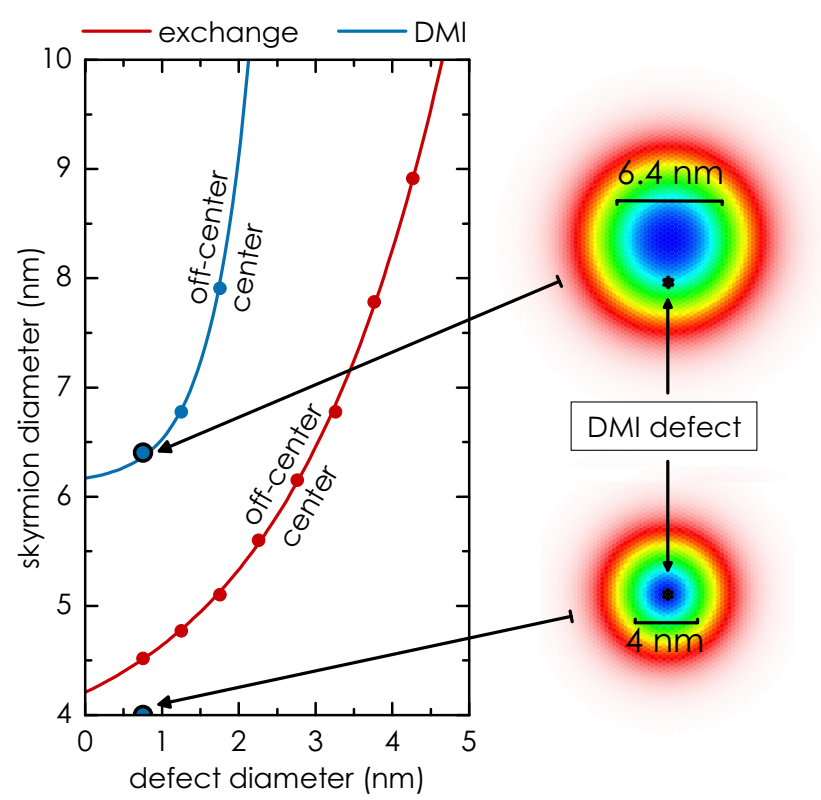

FIG. 4. Pinning regimes (centered or off-centered) of an individual skyrmion at defects induced by variations in either exchange or DMI. Images on the right illustrate skyrmion pinning for considered DMI defects (in black) for $H=0 \mathrm{mT}$ (bottom) and $H=300 \mathrm{mT}$ (top). 

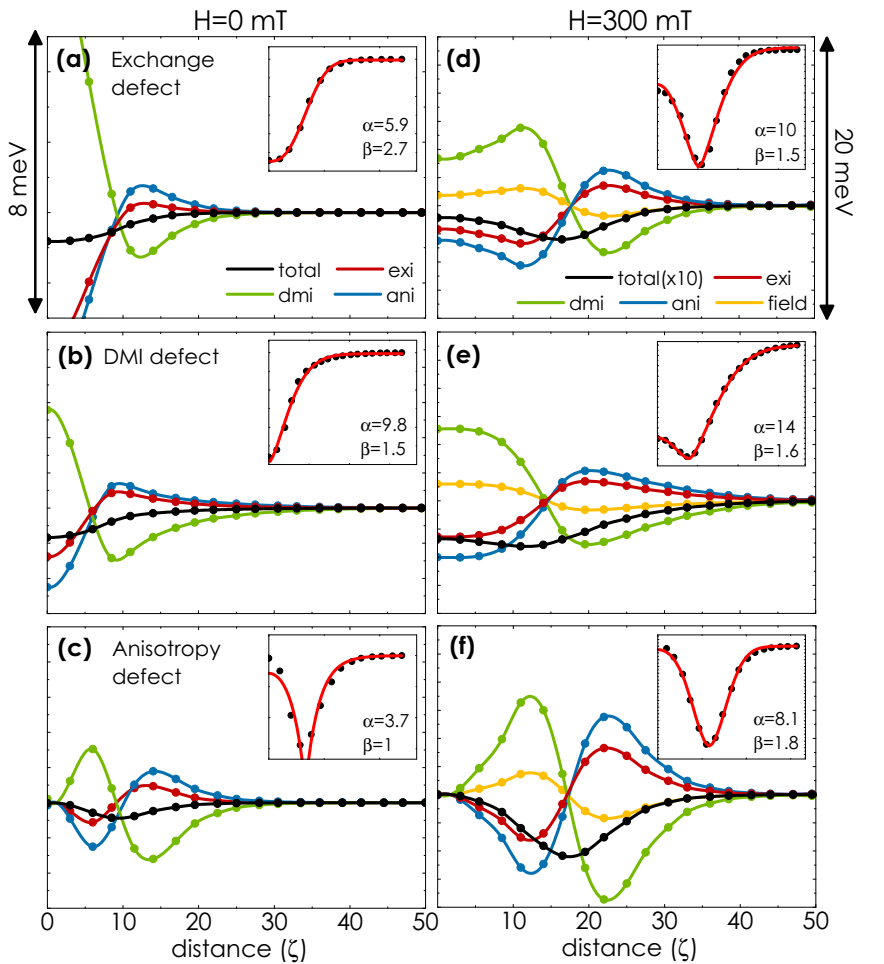

FIG. 5. Separate components of the energy [exchange (exi), Dzyaloshinskii-Moriya (dmi), anisotropy (ani), Zeeman (field)] and the total energy, plotted along the minimum energy paths for skyrmion escape from three kinds of material defects, at $H=0 \mathrm{mT}$ [(a)-(c)], and $H=300 \mathrm{mT}$ [(d)-(f)]. Distances (in units of the lattice constant $\zeta)$ are calculated between the center of the skyrmion core and the center of the defect (placed in the center of the system). Insets plot functional forms of the total energy landscape (i.e., the effective pinning potential) fitted using exponential power function [see Eqs. (2) and (3)].

and without an applied magnetic field at different material defects. The observed behavior of the total energy confirms our previous findings that pinning (position of the energy minimum) occurs at the center for small skyrmions and off-center for large skyrmions, except for anisotropy defects where pinning is always off-center. From the individual energy contributions we find that the transition mechanisms are similar across different material defects and pinning positions. As the skyrmion approaches the defect from a distant point, the exchange and anisotropy energies increase (i.e., become less favorable than when the skyrmion is in an unpinned state), contrary to the behavior of the DMI and Zeeman energies. The individual energy contributions reach a maximum (or minimum) when the defect is close to the domain wall and decrease (or increase) as the skyrmion engulfs the defect. In the case of centered pinning, the described behavior of energy components persists up to the minimum in total energy at the center, whereas for off-center pinning the individual energy terms will peak somewhere between the center and the actual pinning location. This can be summarized as follows: while in terms of exchange and anisotropy energy the defect acts as an obstacle, adding to the skyrmion energy once it is overcome, the total energy remains a potential well due to the apparent dominance of DMI.

The effective pinning potential is often assumed to be parabolic in phenomenological models [28-30]. As seen in Fig. 5, the situation is much more complex, with pinning potentials that either capture the skyrmion in a well centered in the defect, or those that lead to off-center pinning locations. The insets in Fig. 5 reveal that potentials for centered pinning are well represented by an exponential power function (or generalized Gaussian) of the form

$$
\mathcal{G}(\rho) \propto-e^{-(\rho / \alpha)^{\beta}},
$$

where $\rho$ is the distance of the center of the skyrmion to the center of the defect (in units of $\zeta$ ), and $\alpha$ and $\beta$ are the scale and the shape parameter, respectively. The off-center pinning potentials are reasonably well described by the same function, shifted by the radial coordinate $R$ at which the skyrmion is pinned (exactly at, or near the domain wall of the skyrmion; cf. Table I):

$$
\mathcal{G}(\rho) \propto-e^{-(|\rho-R| / \alpha)^{\beta}} .
$$

The coefficients best describing the pinning potentials for variations in material parameters that induce inlayer defects in Fig. 5 are listed in the corresponding insets. We note that the functional dependence of Eqs. (2) and (3) is preserved even in the presence of the magnetic field. It is obvious that coefficients $\alpha$ and $\beta$ depend on all involved parameters, but the detailed description of that dependence is beyond the scope of this work.

\section{B. Pinning in holes}

As the last type of possible defects in a monolayer $\mathrm{Co}$ on $\mathrm{Pt}(111)$, we consider the physical hole due to vacancies in the magnetic layer. Such a defect can be modeled by removing a set of lattice sites and the corresponding bonds (see Fig. 2). In this case, any bond involving a missing atom must be excluded, as opposed to the case of material defects where only bonds between pairs of defect atoms were affected. Minimum energy paths in Figs. 6(a) and 6(b) indicate that the total energy is characterized by a weak energy barrier when transitioning from an unpinned to a pinned state as skyrmion spins are pressed against the edge of the hole before the skyrmion engulfs the pinning center, while individual energy contributions behave in a similar fashion to previously discussed cases of material defects. Furthermore, Fig. 6(c) shows that the preferable position of the skyrmion can be either at the center or at the domain wall (off-center), depending on the ratio of skyrmion to hole size.

Therefore, the effective pinning potential for skyrmions in the case of holes in the magnetic material is somewhat more complex as described by Eqs. (2) and (3), and needs to be expanded by a decaying exponential in order to describe the energy barrier that skyrmion needs to overcome before resting into the potential well at the pinning site:

$$
\mathcal{G}(\rho) \propto-e^{-(|\rho-R| / \alpha)^{\beta}}+A e^{-\rho / \gamma} .
$$

Here $\rho$ is the distance of the center of the skyrmion to the center of the defect (in units of $\zeta$ ), $\alpha$ and $\beta$ are the scale and the shape parameter [as in Eq. (3)], $R$ is either zero or equal 

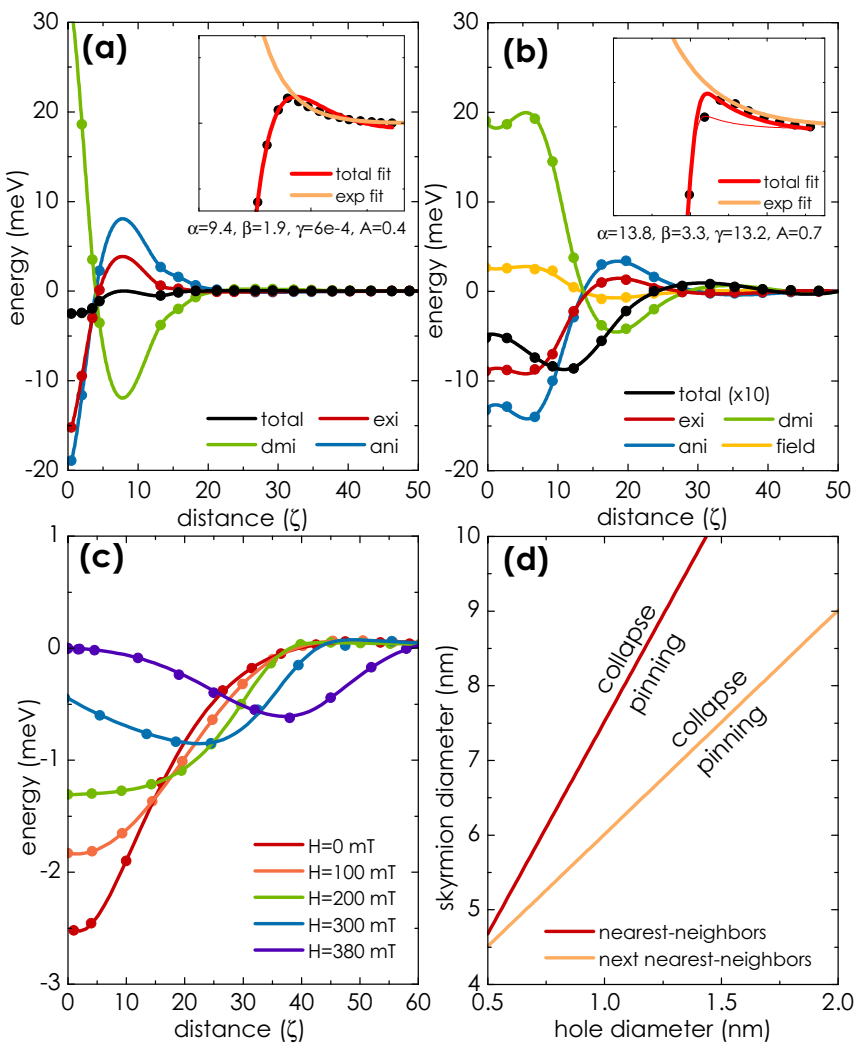

FIG. 6. Pinning of a skyrmion at a hole in Co monolayer on Pt. Separate components and the total energy are plotted along the minimum energy paths of the skyrmion pinned to a hole, for (a) $H=0 \mathrm{mT}$, and (b) $H=300 \mathrm{mT}$. Distances (in units of the lattice constant $\zeta$ ) are calculated between the center of the skyrmion core and the center of the hole. Insets plot the effective pinning potential fitted using Eq. (4). (c) Evolution of the pinning potential of a hole with diameter $0.5 \mathrm{~nm}$, for different applied magnetic fields. (d) The boundary between regimes of skyrmion pinning and skyrmion collapse at $H=0$, as a function of the skyrmion and defect size, for nearest- and next-nearest-neighbor approximations of the exchange interaction.

to the radius of the domain wall of the skyrmion, and $\gamma$ is the exponential decay constant for the relaxation of the skyrmion far away from the hole. The coefficients best describing the pinning potentials in the presence of a hole in Figs. 6(a) and 6(b) are listed in the corresponding insets. Figure 6(c) shows that the functional form in Eq. (4) accurately describes the pinning potentials for different values of the magnetic field, i.e., different size of the skyrmion, with coefficients depending on all involved parameters (including the size of the hole and the magnetic field).

However, the presence of a hole can also be detrimental for the skyrmions, as the skyrmion may isotropically collapse (magnetic spins undergo collective rotation in the radial direction toward the uniform FM state) for sufficiently large defects. Figure $6(\mathrm{~d})$ reveals that small vacancies in the magnetic material, which make up only $1 \%$ of the skyrmion core, can already induce a collapse of the skyrmion. This is likely due to the low activation barriers needed to stabilize individual skyrmions in a Co monolayer on Pt. For example, a defect with three atomic vacancies ( 24 missing bonds) will

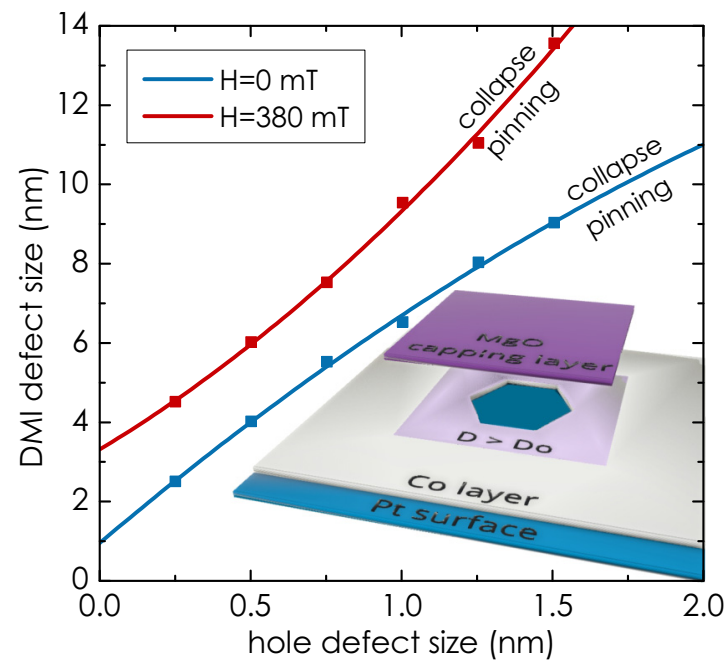

FIG. 7. Skyrmion pinning at a hole in the Co monolayer on $\mathrm{Pt}$, surrounded by a region with higher DMI due to the $\mathrm{MgO}$ capping layer. Regimes of skyrmion pinning and collapse as described in Fig. 6, using the next-nearest-neighbor exchange approximation, in the absence and presence of an external magnetic field. Inset: Artistic illustration of the considered structure.

induce a collapse of the $4 \mathrm{~nm}$ skyrmion, since the amount of energy lost in the defect essentially removes the energy barrier for isotropic collapse, of a mere $36 \mathrm{meV}$. Therefore, the collapse of an isolated skyrmion is facilitated by the presence of magnetic vacancies, which makes its stability much more sensitive than previously anticipated. Similar findings of poor skyrmion stability were reported by Ref. [65], and were found to be in agreement with experimental measurements involving skyrmion nucleation near structural defects [3].

Since this poor stability around the defect in our simulations could be an artifact of nearest-neighbor approximations, we also tested the influence of the next-nearest-neighbor exchange interactions $\left(J_{1}=29 \mathrm{meV}\right.$ per bond and $J_{2}=-0.6 \mathrm{meV}$ per bond). Similar results are obtained [see Fig. 6(d)], albeit with somewhat weaker scaling since the antiferromagnetic exchange improves skyrmion stability. While monolayer skyrmions (typically less than $10 \mathrm{~nm}$ in diameter) will annihilate for defects larger than $1.5 \mathrm{~nm}$, room-temperature skyrmions that have been recently observed, with size between 30 and $100 \mathrm{~nm}$ [13-15], will pin and survive for up to 5 and $8.5 \mathrm{~nm}$ large holes, respectively. Although Ref. [30] reported stable skyrmions near holes in the magnetic layer, only a single missing spin was considered there, and micromagnetic simulations could not properly capture the skyrmion annihilation arising from a Bloch point.

A possible way to improve skyrmion stability in the presence of a hole involves adding a heavy-metal capping layer, i.e. increasing DMI in the vicinity of the hole, as illustrated in the inset of Fig. 7. By increasing the DMI around the hole, the chiral interactions are strengthened and the size of the skyrmion increases, which permits the presence of larger holes in the material without harming the skyrmion stability. Figure 7 shows that individual skyrmions can pin near few-nanometer large holes when using a $\mathrm{MgO}$ capping patch on the Co film on $\mathrm{Pt}\left(D_{i j}=2.23 \mathrm{meV}\right.$ per bond). The patch 


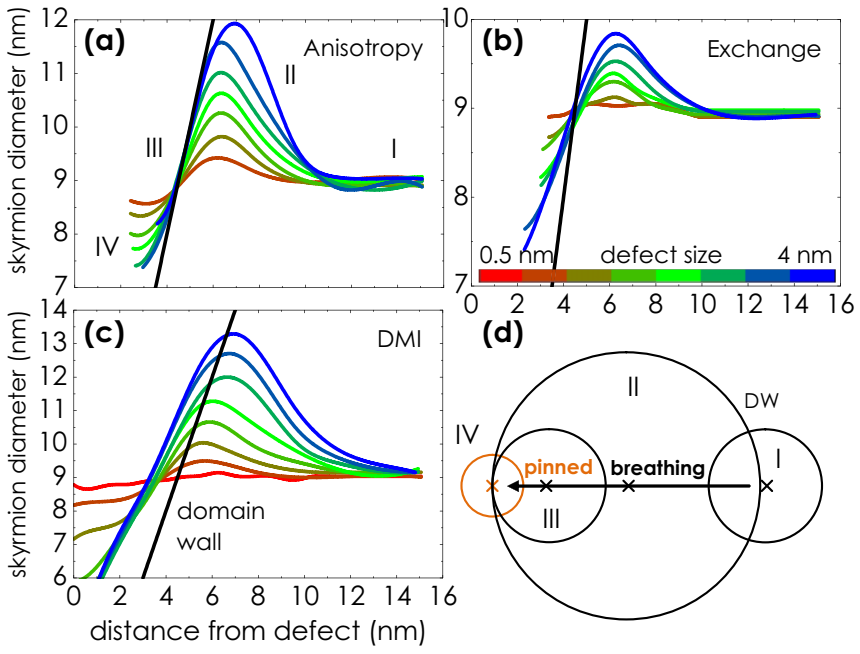

FIG. 8. Breathing of the skyrmion in the vicinity of a defect [for a defect arising from variations in anisotropy (a), exchange (b), and DMI (c)]. Defect diameter is varied between 0.5 and $4 \mathrm{~nm}$ (see color bar) and the applied field was held at $H=380 \mathrm{mT}$. In each panel the solid black line denotes the position where the domain wall of the skyrmion reaches the defect. (d) Schematic illustration explaining the observed breathing behavior (solid line depicts the domain wall of the skyrmion): (I) initial skyrmion near a defect, (II) skyrmion expansion, (III) skyrmion shrinking, and (IV) pinned skyrmion.

needed to prevent skyrmion annihilation in the present case is more than an order of magnitude larger than the hole, but can be designed smaller by using a heavy metal that induces a stronger DMI in the film than $\mathrm{MgO}$ does.

\section{EMPLOYING THE DEFECTS}

Defects were shown to exert pinning and thus affect dynamics of magnetic skyrmions. It is therefore of interest to envisage possible use of defects in existing and emergent spintronic devices. In this section we consider three distinct defect geometries which can be exploited to induce particular behavior of skyrmions. Needed defects (or periodic [34,35] and random [36-38] arrays thereof) can be constructed by spatial engineering of relevant parameters by, e.g., patterning an additional (e.g., heavy-metal) layer (MgO, Ir, or other) on top of the $\mathrm{Co} / \mathrm{Pt}$ system in the desired geometry of the defect.

\section{A. Controlled breathing of a skyrmion}

The interaction of an isolated skyrmion with a defect can affect skyrmion motion [29,30], in a nontrivial fashion and in a plethora of ways. One can also expect that variations in size and other skyrmion properties should arise due to the spatially inhomogeneous pinning forces. Figure 8 reveals that defects induce a breathing behavior on skyrmions: the skyrmion expands as it approaches a defect and then shrinks back to its original size at the pinning position. While the maximal bloat of a skyrmion depends on the defect size, the pinning position and size recovery point will instead rely on the type of the defect. For example, one can see from Fig. 8 that the skyrmion diameter recovers its unpinned value

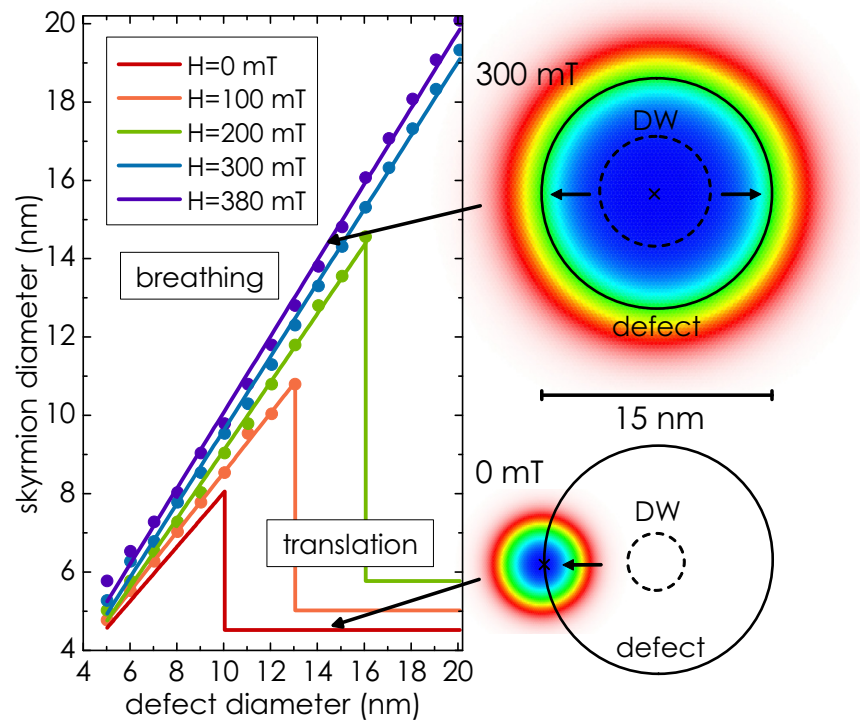

FIG. 9. Skyrmion behavior in a defect with inhomogeneous DMI shaped as a ring, as a function of ring diameter and for different applied magnetic fields. Illustration on the right depicts a skyrmion either expanding to overlap its domain wall with the ring defect (top) or translating so that its core locates at the defect (bottom). The defect is shown by a solid line, while the dotted line corresponds to the domain wall (DW) of the initial skyrmion.

when the domain wall sits on the defect for anisotropic and exchange defects, but for the defect between the domain wall and the center of the skyrmion for defects arising from DMI inhomogeneity. Figure 8(d) depicts a schematic explanation for this breathing behavior. At a given distance from the defect (I) the skyrmion favors energetically that its domain wall [shown by a solid line in Fig. 8(d)] sits at the pinning center, and thus expands to reach such configuration (II), to subsequently shrink once approaching the defect (III). For the case of an off-center pinning, the skyrmion recovers its unpinned size after stage III, whereas in the case of centered pinning the diameter of the skyrmion is further reduced when its core occupies the pinning site (IV). Note that the skyrmion can easily experience substantial variations in diameter (by as much as $50 \%$ ) for relatively large defects.

Clearly, the pinning influence of material defects on isolated skyrmions can be used to control the skyrmion diameter. To optimize such manipulation, we consider defects that are aligned to form rings, matching the oval shape of the skyrmion. Such defects can be induced through variations in DMI, where a heavy metal is patterned in the desired shape on top of the magnetic layer. Figure 9 shows that when a skyrmion is placed inside the ring defect it can either translate so that it remains centered on the ring, or expand until the domain wall reaches the preferred position by coinciding with the ring defect. For centered pinning the skyrmion retains its original diameter, whereas in the case of off-center pinning the skyrmion diameter will match that of the defect. Figure 9 also shows that the dynamics of the skyrmion (translation or expansion) depends on the ratio of the size of the unpinned skyrmion (dotted line) to the defect size. Breathing of the skyrmion only occurs for small defects $(<10 \mathrm{~nm})$ when no 

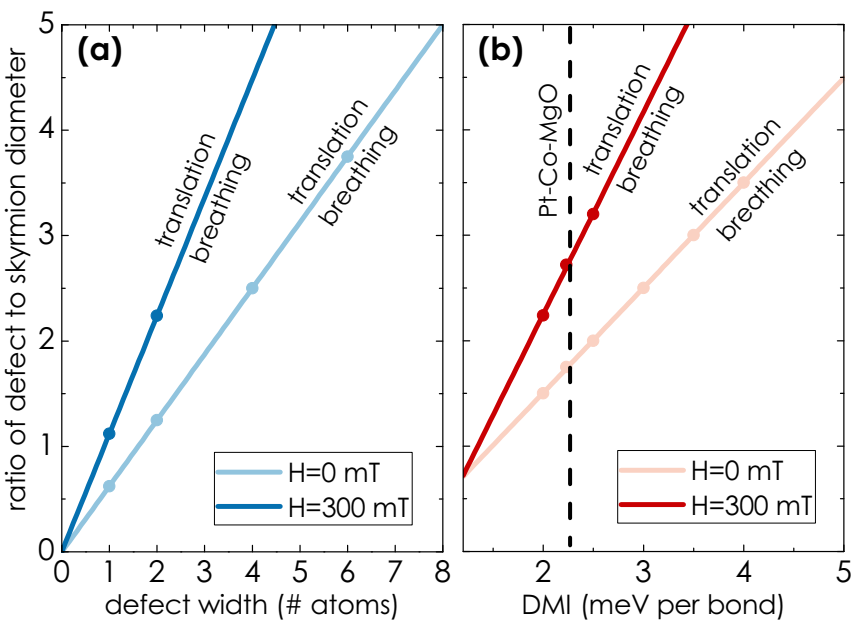

FIG. 10. Pinning regimes (either skyrmion expansion or translation) in the case of a ring defect, for different ratios of defect to skyrmion diameters, as a function of (a) the width of the defect, and (b) DMI strength.

magnetic field is applied, but remains present in larger defects (even above $20 \mathrm{~nm}$ ) for higher external fields. Figure 10 shows that the transition line scales with both the width of the defect ring and the DMI strength. Increasing the width of the ring enables skyrmions to grow two to four times their size in order to reach more stable configurations. For known parameters of realistic material defects, such as a $\mathrm{MgO}$ capping layer patterned in the desired shape on Co film on $\mathrm{Pt}$, we have realized the expansion of the skyrmion by defects that are several times the original size of the skyrmion, depending on the external magnetic field. This efficient manipulation of the skyrmion size suggests particular applications, for example in optics, based on the interaction of polarized light [66] with preformed periodic spin structures such as pinned skyrmion arrays, or in skyrmion-based magnonic crystals [67] tailored at will by the nanoengineered pinning.

\section{B. Skyrmion ratchet}

Ratcheting of particles in asymmetric potentials, and thereby creating a net motion in one direction despite a symmetric drive, is of pertinent interest in biological systems and pharmaceutical applications. A similar principle has already been employed to other, seemingly very remote systems, such as vortices in superconductors. There, vortices are carriers of dissipation in the presence of applied current, and asymmetric pinning potentials have been employed to either remove vortices from the sample in the presence of an ac drive, or to broadly manipulate the vortex motion and engineer fluxonic devices. For possible spintronic devices based on skyrmions, one therefore also wonders to which extent the analogy to the above systems can be applied.

For example, for a row of triangular holes in the superconducting film and an applied ac current as shown in Fig. 11(a), the vortex will oscillate perpendicular to the direction of the current, but will experience an asymmetric depinning force in up/down directions due to the triangular shape of the pinning site. This results in a net vertical movement of

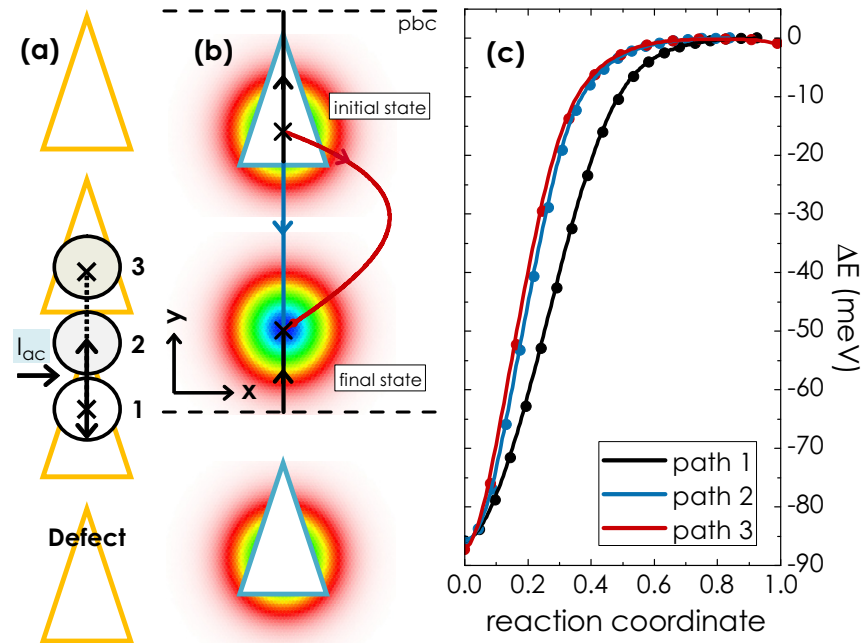

FIG. 11. Skyrmion motion along a linear row of planar triangular defects induced by changes in DMI. (a) Schematic illustration of the considered defect geometry. (b) Spatially resolved paths for escape of the skyrmion from the defect: along the upper apex (in black), across the base (in blue), and over the side edge (in red). (c) Energy differences of the three paths in (b) with respect to the initial (pinned) state.

the vortex (dotted line) along the nanostructured pinning. A similar process could be devised to control the motion of magnetic skyrmions, as an ac current-based method for controlling skyrmion positions and motion for spintronic applications.

To examine this, we calculated the interaction of a skyrmion with the triangular defect created by stronger DMI, $D=2 D_{0}$, as seen in Fig. 11(b). Figure 11(b) shows three possible paths the skyrmion can take in order to escape from such a triangular defect: over its protruded apex, on the lateral side, or over the base of the triangular defect. Minimum energy paths reveal that the path toward the top apex of the triangle is, in fact, much lower in energy than the remaining two paths [see Fig. 11(c)], with the barrier for skyrmion depinning being higher on the side of the triangle, and highest at its base. As a result, for a prepatterned line of triangular defects as shown in Fig. 11(a), in the presence of an ac drive for skyrmions, or even for sufficiently large thermal fluctuations, a skyrmion will exhibit a net motion in the bottom-to-top direction, due to the asymmetry in the depinning potential seen in Fig. 11(c). Our simulations thereby prove the ratchet concept for skyrmions in the presence of asymmetric defects, but more detailed dynamic simulations are needed to optimize the performance of such a ratchet and skyrmion guidance, bearing in mind the peculiarities of the Magnus force and the resulting skyrmion orbits [68]. Due to such differences from other previously considered ratcheting systems, it is of fundamental value to further examine a possible variety of skyrmion ratchet behaviors in nanoengineered heterochiral systems, the use of asymmetric ac driving [39], as well as the novel collective effects due to the competition of skyrmion-skyrmion interactions (possibly even nonmonotonic [69]) with a given geometry of a preformed pinning lattice. 

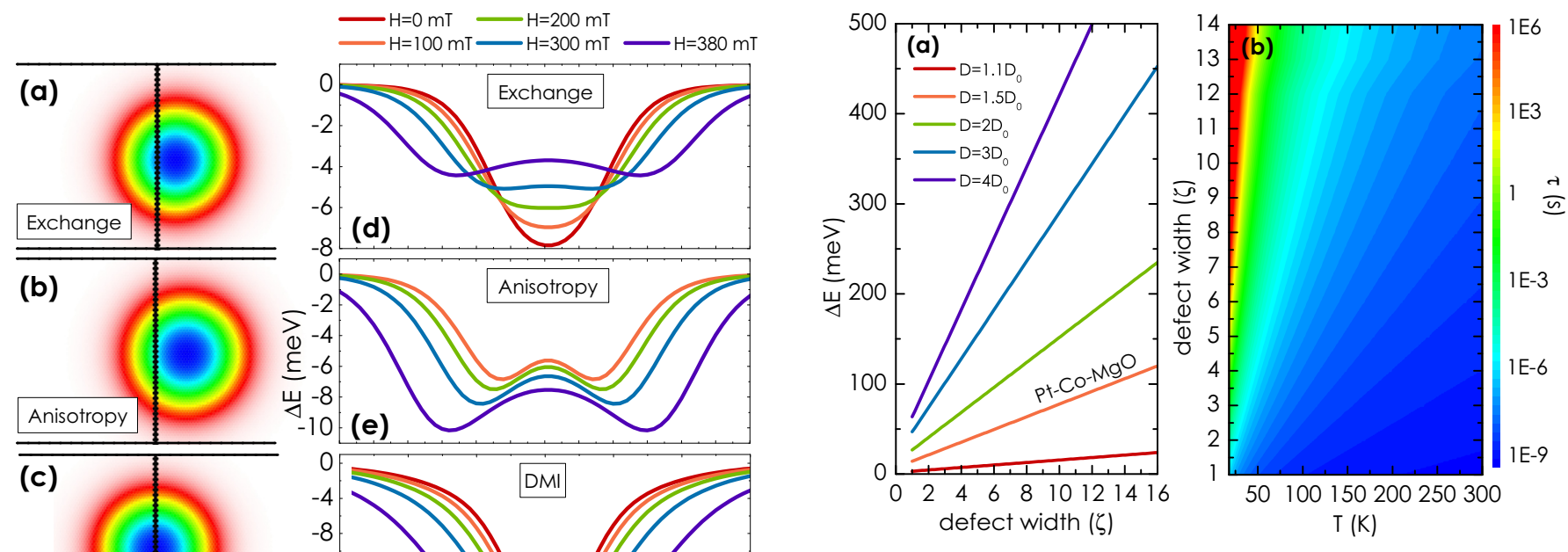

FIG. 13. Depinning a skyrmion from a linear defect with stronger DMI. (a) Energy barrier for depinning as a function of defect width and DMI strength there. (b) Skyrmion "lifetime" in the racetrack for various temperatures and widths of the nanoengineered pinning trail, ranging from nanoseconds (blue) to days (red).

FIG. 12. Skyrmion pinning at a linear defect, to be used in racetrack memories. (a)-(c) Preferential pinning positions on the considered defects (with variations in either exchange, anisotropy, or DMI). (d)-(f) Energy difference as a function of the position of the skyrmion with respect to the defect, obtained by minimum energy path calculations.

\section{Skyrmion on the rails}

Since we have focused most of our analysis so far on point-localized defects, we next discuss the pinning behavior of skyrmions on extended defects where material properties are changed. We first consider the motion of an isolated skyrmion along a linear defect [40-43] (a narrow strip with a varied material parameter) to be used in, e.g., racetrack memories [see Figs. 12(a)-12(c)]. Figure 12 reveals that exchange defect supports either center or off-center pinning of skyrmions, while anisotropy defects only pin off-center, as expected from our earlier findings. On the other hand, linear defects induced by varied DMI can only pin a skyrmion at its center, which differs from point defects where pinning could occur either at the center or at an off-center locus. These results suggest that for linear defects, variations in DMI will produce more stable pinning, being at the center of the skyrmion core, and with significantly higher energy barrier than for off-center pinning. Figure 13(a) shows that the energy barriers for depinning a skyrmion scale linearly with the width of the linear DMI defect, while the slope of that scaled behavior increases with the DMI strength inside the defect. For the case of the $\mathrm{MgO}$ capping layer patterned on monolayer Co on Pt substrate, we find that the energy required to depin the skyrmion surpasses that needed for the skyrmion to self-collapse, which was found to be $37 \mathrm{meV}$ in Ref. [48]. We can also quantify the "lifetime" of the pinned state (that is, the time at which the skyrmion depins from the defect in the presence of fluctuations) using the Arrhenius law $\tau=\tau_{0} \exp \left(\Delta E / k_{B} T\right)$, where $\tau_{0}$ is related to the attempt frequency $f_{0}=1 / \tau_{0}, \Delta E$ is the energy barrier, $k_{B}$ is the Boltzmann constant, and $T$ is the temperature. In these estimates, the attempt frequencies are taken to be $f_{0}=10 \mathrm{GHz}$

as suggested in Ref. [47]. Figure 13(b) reveals that the pinning lifetimes are in the nanosecond regime for room temperatures, as expected for monolayer skyrmions, but can be in the order of seconds at lower temperatures. While the pinned state for thin defects $(\leqslant 5 \zeta)$ can only survive milliseconds and at very low temperatures, seconds-long lifetimes can be achieved at temperatures above $100 \mathrm{~K}$ when increasing the defect

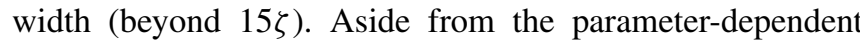
quantitative analysis, our findings clearly show that thermal stability of isolated skyrmions in racetrack memories can be significantly improved by pinning the skyrmion on linear defects, which can be also combined with the recent proposal of advanced heterochiral racetracks with greatly increased barrier for detrimental skyrmion exit from the track [57,63], to reach the optimal conditions for skyrmion stability in applied systems with finite geometries [60]. Lastly, we note that very similar considerations can be applied to the linear defects (besides the DMI ones) where off-center pinning of skyrmions is favorable - but then using two parallel linear defects that optimally pin the skyrmion on both sides off the center of its core, as a true "skyrmion on the rails."

\section{CONCLUSIONS}

In summary, we have reported the fundamental properties of chiral ferromagnetic systems related to pinning of isolated skyrmions, taking Co monolayer on $\mathrm{Pt}$ as an exemplary system. Our consideration of different atomic defects (including local variations in exchange, anisotropy, DMI, and vacancies in the material) yields a more complete understanding of how magnetic skyrmions pin to defects. In particular, we find that pinning can occur at the skyrmion center, domain wall, or in-between the two, depending on the type of defect being used and the ratio of skyrmion core to defect size. The effective pinning potentials have complex functional forms that cannot be described by parabolic shapes. Surprisingly, the collapse of skyrmions is strongly facilitated by the 
presence of atomic-scale vacancies, which indicates a much weaker stability of skyrmions than previously anticipated. One possible solution to improve skyrmion stability involves increasing the DMI (e.g., by a heavy-metal capping layer) in the vicinity of vacancies/holes in the magnetic material. We also demonstrated that the interaction of a skyrmion with a defect leads to a "breathing" behavior of skyrmion core on its path to the pinning site: the skyrmion expands as it approaches a defect and then shrinks back to its original size at the pinning position. Even in a stationary regime, the size of the skyrmion core can be very broadly controlled by the size of the pinning site, as we demonstrated on the example of a prepatterned ringlike defect that stretches the domain wall of the skyrmion. Finally, we discussed the cases of asymmetric and linearly extended defects, that further enable the use of our findings in the design of next-generation spintronic devices, such as those based on interaction with polarized light, tunable magnonic crystals, skyrmion ratchet systems, and racetrack memories. Since spatial engineering of defects in a ferromagnetic film is within experimental reach [for example, by targeted deposition of a (nanostructured) heavy-metal layer, such as Ir or $\mathrm{MgO}$ ], we expect that our considerations will indeed facilitate efficient manipulation of skyrmions in practical applications.

\section{ACKNOWLEDGMENTS}

This work was supported by the Research Foundation, Flanders (FWO-Vlaanderen) and Brazilian agency $\mathrm{CNPq}$ (Grants No. 442668/2014-7 and No. 140840/2016-8).
[1] A. N. Bogdanov and U. K. Rössler, Phys. Rev. Lett. 87, 037203 (2001).

[2] U. K. Rössler, A. N. Bogdanov, and C. Pfleiderer, Nature (London) 442, 797 (2006).

[3] N. Romming, C. Hanneken, M. Menzel, J. E. Bickel, B. Wolter, K. von Bergmann, A. Kubetzka, and R. Wiesendanger, Science 341, 636 (2013).

[4] S. Mühlbauer, B. Binz, F. Jonietz, C. Pfleiderer, A. Rosch, A. Neubauer, R. Georgii, and P. Böni, Science 323, 915 (2009).

[5] C. Pappas, E. Lelièvre-Berna, P. Falus, P. M. Bentley, E. Moskvin, S. Grigoriev, P. Fouquet, and B. Farago, Phys. Rev. Lett. 102, 197202 (2009).

[6] E. Moskvin, S. Grigoriev, V. Dyadkin, H. Eckerlebe, M. Baenitz, M. Schmidt, and H. Wilhelm, Phys. Rev. Lett. 110, 077207 (2013).

[7] S. Heinze, K. von Bergmann, M. Menzel, J. Brede, A. Kubetzka, R. Wiesendanger, G. Bihlmayer, and S. Blügel, Nat. Phys. 7, 713 (2011).

[8] I. Dzyaloshinsky, J. Phys. Chem. Solids 4, 241 (1958).

[9] T. Moriya, Phys. Rev. 120, 91 (1960).

[10] J. Sampaio, V. Cros, S. Rohart, A. Thiaville, and A. Fert, Nat. Nanotechnol. 8, 839 (2013).

[11] N. Romming, A. Kubetzka, C. Hanneken, K. von Bergmann, and R. Wiesendanger, Phys. Rev. Lett. 114, 177203 (2015).

[12] A. Bogdanov and A. Hubert, J. Magn. Magn. Mater. 138, 255 (1994).

[13] S. Woo, K. Litzius, B. Krüger, M.-Y. Im, L. Caretta, K. Richter, M. Mann, A. Krone, R. M. Reeve, M. Weigand, P. Agrawal, I. Lemesh, M.-A. Mawass, P. Fischer, M. Kläui, and G. S. D. Beach, Nat. Mater. 15, 501 (2016).

[14] C. Moreau-Luchaire, C. Moutafis, N. Reyren, J. Sampaio, C. A. F. Vaz, N. Van Horne, K. Bouzehouane, C. Garcia, K. Deranlot, P. Warnicke, P. Wohlhüter, J.-M. George, M. Weigand, J. Raabe, V. Cros, and A. Fert, Nat. Nanotechnol. 11, 444 (2016).

[15] O. Boulle, J. Vogel, H. Yang, S. Pizzini, D. S. de Souza Chaves, A. Locatelli, T. O. Mentes, A. Sala, L. D. Buda-Prejbeanu, O. Klein, M. Belmeguenai, Y. Roussigné, Y. A. Stashkevich, S. M. Chérif, L. Aballe, M. Foerster, M. Chshiev, S. Auffret, I. M. Miron, and G. Gaudin, Nat. Nanotechnol. 11, 449 (2016).

[16] R. Wiesendanger, Nature Reviews Materials 1, 16044 (2016).

[17] F. Jonietz, S. Mühlbauer, C. Pfleiderer, A. Neubauer, W. Münzer,
A. Bauer, T. Adams, R. Georgii, P. Böni, R. A. Duine, K. Everschor, M. Garst, and A. Rosch, Science 330, 1648 (2010).

[18] J. Iwasaki, M. Mochizuki, and N. Nagaosa, Nat. Commun. 4, 1463 (2013).

[19] N. S. Kiselev, A. N. Bogdanov, R. Schäfer, and U. K. Rössler, J. Phys. D 44, 392001 (2011).

[20] A. Fert, V. Cros, and J. Sampaio, Nat. Nanotechnol. 8, 152 (2013).

[21] X. Zhang, M. Ezawa, and Y. Zhou, Sci. Rep. 5, 9400 (2015).

[22] D. Liang, M. J. Stolt, and S. Jin, Nat. Phys. 12, 25 (2016).

[23] S. Krause and R. Wiesendanger, Nat. Mater. 15, 493 (2016).

[24] C. Hanneken, F. Otte, A. Kubetzka, B. Dupé, N. Romming, K. von Bergmann, R. Wiesendanger, and S. Heinze, Nat. Nanotechnol. 10, 1039 (2015).

[25] S. S. P. Parkin, M. Hayashi, and L. Thomas, Science 320, 190 (2008).

[26] T. Schulz, R. Ritz, A. Bauer, M. Halder, C. Wagner, M. Franz, C. Pfleiderer, M. Everschor, K. Garst, and A. Rosch, Nat. Phys. 8, 301 (2012).

[27] X. Z. Yu, N. Kanazawa, W. Z. Zhang, T. Nagai, T. Hara, K. Kimoto, Y. Matsui, Y. Onose, and Y. Tokura, Nat. Commun. 3, 988 (2012).

[28] S.-Z. Lin, C. Reichhardt, C. D. Batista, and A. Saxena, Phys. Rev. B 87, 214419 (2013).

[29] Y.-H. Liu and Y.-Q. Li, J. Phys.: Condens. Matter 25, 076005 (2013).

[30] J. Müller and A. Rosch, Phys. Rev. B 91, 054410 (2015).

[31] J.-V. Kim and M.-W. Yoo, Appl. Phys. Lett. 110, 132404 (2017).

[32] W. Legrand, D. Maccariello, N. Reyren, K. Garcia, C. Moutafis, C. Moreau-Luchaire, S. Collin, K. Bouzehouane, V. Cros, and A. Fert, Nano Lett. 17, 2703 (2017).

[33] C. Hanneken, K. Kubetzka, A. von Bergmann, and R. Wiesendanger, New J. Phys. 18, 055009 (2016).

[34] F. Ma, C. Reichhardt, W. Gan, C. J. Olson Reichhardt, and W. S. Lew, Phys. Rev. B 94, 144405 (2016).

[35] C. Reichhardt, D. Ray, and C. J. Olson Reichhardt, Phys. Rev. B 91, 104426 (2015).

[36] C. Reichhardt, D. Ray, and C. J. Olson Reichhardt, Phys. Rev. Lett. 114, 217202 (2015).

[37] S. A. Díaz, C. J. O. Reichhardt, D. P. Arovas, A. Saxena, and C. Reichhardt, Phys. Rev. B 96, 085106 (2017). 
[38] E. M. Chudnovsky and D. A. Garanin, Skyrmion glass in a disordered magnetic film, arXiv:1710.10608.

[39] X. Ma, C. J. Olson Reichhardt, and C. Reichhardt, Phys. Rev. B 95, 104401 (2017).

[40] C. Reichhardt and C. J. Olson Reichhardt, Phys. Rev. B 95, 014412 (2017).

[41] C. Navau, N. Del-Valle, and A. Sanchez, Phys. Rev. B 94, 184104 (2016).

[42] C. Reichhardt and C. J. Olson Reichhardt, Phys. Rev. B 94, 094413 (2016).

[43] C. Reichhardt and C. J. Olson Reichhardt, Phys. Rev. B 92, 224432 (2015).

[44] E. Lundgren, B. Stanka, M. Schmid, and P. Varga, Phys. Rev. B 62, 2843 (2000).

[45] U. Pustogowa, J. Zabloudil, C. Uiberacker, C. Blaas, P. Weinberger, L. Szunyogh, and C. Sommers, Phys. Rev. B 60, 414 (1999).

[46] A. Fert and P. M. Levy, Phys. Rev. Lett. 44, 1538 (1980).

[47] S. Rohart, J. Miltat, and A. Thiaville, Phys. Rev. B 93, 214412 (2016).

[48] I. S. Lobanov, H. Jónsson, and V. M. Uzdin, Phys. Rev. B 94, 174418 (2016).

[49] H. Jónsson, G. Mills, and K. W. Jacobsen, Classical and Quantum Dynamics in Condensed Phase Simulations (World Scientific, Singapore, 2011), pp. 385-404.

[50] G. Henkelman, B. P. Uberuaga, and H. Jónsson, J. Chem. Phys. 113, 9901 (2000).

[51] G. Henkelman and H. Jónsson, J. Chem. Phys. 113, 9978 (2000).

[52] Weinan E, Weiqing Ren, and Eric Vanden-Eijnden, Phys. Rev. B 66, 052301 (2002).

[53] S. A. Trygubenko and D. J. Wales, J. Chem. Phys. 120, 2082 (2004).
[54] P. F. Bessarab, V. M. Uzdin, and H. Jónsson, Comput. Phys. Commun. 196, 335 (2015).

[55] R. Dittrich, T. Schrefl, D. Suess, W. Scholz, H. Forster, and J. Fidler, J. Magn. Magn. Mater. 250, 12 (2002).

[56] D. Suess, T. Schrefl, D. Suess, S. Fähle, M. Kirschner, G. Hrkac, F. Dorfbauer, and J. Fidler, Appl. Phys. Lett. 87, 012504 (2005).

[57] D. Stosic, J. Mulkers, B. Van Waeyenberge, T. B. Ludermir, and M. V. Milošević, Phys. Rev. B 95, 214418 (2017).

[58] P. F. Bessarab, G. P. Müller, I. S. Lobanov, F. N. Rybakov, N. S. Kiselev, H. Jónsson, V. M. Uzdin, S. Blügel, L. Bergqvist, and A. Delin, Lifetime of racetrack skyrmions, arXiv:1706.07173 .

[59] P. F. Bessarab, Phys. Rev. B 95, 136401 (2017).

[60] D. Cortés-Ortuño, W. Wang, M. Beg, R. A. Pepper, M.-A. Bisotti, R. Carey, M. Vousden, T. Kluyver, O. Hovorka, and H. Fangohr, Sci. Rep. 7, 4060 (2017).

[61] D. Sheppard, R. Terrell, and G. Henkelman, J. Chem. Phys. 128, 134106 (2008).

[62] J. Zang, M. Mostovoy, J. H. Han, and N. Nagaosa, Phys. Rev. Lett. 107, 136804 (2011).

[63] J. Mulkers, B. Van Waeyenberge, and M. V. Milošević, Phys. Rev. B 95, 144401 (2017).

[64] K. Everschor, M. Garst, B. Binz, F. Jonietz, S. Mühlbauer, C. Pfleiderer, and A. Rosch, Phys. Rev. B 86, 054432 (2012).

[65] V. M. Uzdin, M. N. Potkina, I. S. Lobanov, P. F. Bessarab, and H. Jónsson, Physica B: Condens. Matter (2017), doi:10.1016/j.physb.2017.09.040.

[66] N. Ogawa, S. Seki, and Y. Tokura, Sci. Rep. 5, 9552 (2015).

[67] F. Ma, Y. Zhou, H. B. Braun, and W. S. Lew, Nano Lett. 15, 4029 (2015).

[68] C. Reichhardt, D. Ray, and C. J. O. Reichhardt, New J. Phys. 17, 073034 (2015).

[69] L. Rózsa, A. Deák, E. Simon, R. Yanes, L. Udvardi, L. Szunyogh, and U. Nowak, Phys. Rev. Lett. 117, 157205 (2016). 